\title{
Noise Reduction for Variance-Based Device-Free Localization and Tracking
}

\author{
Yang Zhao and Neal Patwari
}

\begin{abstract}
Human motion in the vicinity of a wireless link causes variations in the link received signal strength (RSS). Device-free localization (DFL) systems, such as variance-based radio tomographic imaging (VRTI) use these RSS variations in a wireless network to detect, locate and track people in the area of the network, even through walls. However, intrinsic motion, such as branches moving in the wind, rotating or vibrating machinery, also causes RSS variations which degrade the performance of a DFL system. In this paper, we propose and evaluate a subspace decomposition method subspace variance-based radio tomography (SubVRT) to reduce the impact of the variations caused by intrinsic motion. Experimental results show that the SubVRT algorithm reduces localization root mean squared error (RMSE) by $41 \%$. In addition, the Kalman filter tracking results from SubVRT have $97 \%$ of errors less than $1.4 \mathrm{~m}$, a $65 \%$ improvement compared to tracking results from VRTI.
\end{abstract}

\section{INTRODUCTION}

As an emerging technology, device-free localization (DFL) using radio frequency (RF) sensor networks has potential application in detecting intruders in industrial facilities, and helping police and firefighters track people inside a building during an emergency [1]. In these scenarios, people to be located cannot be expected to participate in the localization system by carrying radio devices, thus standard radio localization techniques are not useful for these applications.

Various RF measurements including ultra-wideband (UWB) and received signal strength (RSS) have been proposed and applied to detect, locate and track people who do not carry radio devices in an indoor environment [2], [3], [4], [5]. RSS measurements are inexpensive and available in standard wireless devices, and have been used in different DFL studies with surprising accuracy [4], [6], [5]. These RSS-based DFL methods essentially use a windowed variance of RSS measured on static links. For example, [5] deploys an RF sensor network around a residential house and uses sample variance during a short window to track people walking inside the house; [6] places RF sensors on the ceiling of a room, and track people using the RSSI dynamic, which is essentially the variance of RSS measurements with and without people moving inside the room. In this paper we use windowed variance to describe the various functions of RSS measurements recently used in different DFL studies [4], [6], [5], [7], and we call these methods RSS variance-based DFL methods.

Y. Zhao and N. Patwari are with the Department of Electrical and Computer Engineering, University of Utah, Salt Lake City, USA. This material is based upon work supported by the National Science Foundation under Grant Nos. \#0748206 and \#1035565. E-mails: yang.zhao@utah.edu and npatwari@ece.utah.edu.
For variance-based DFL methods, variance can be caused by two types of motion: extrinsic motion and intrinsic motion. Extrinsic motion is defined as motion of people and other objects that enter and leave the environment. Intrinsic motion is defined as motion of objects that are intrinsic parts of the environment, objects which cannot be removed without fundamentally altering the environment. If a significant amount of windowed variance is caused by intrinsic motion, then it may be difficult to detect extrinsic motion. For example, rotating fans, leaves and branches swaying in wind, and moving or rotating machines in a factory all may impact the RSS measured on static links. Also, if RF sensors are vibrating or swaying in the wind, their RSS measurements change as a result. Even if the receiver moves by only a fraction of its wavelength, the RSS may vary by several orders of magnitude. We call variance caused by intrinsic motion and extrinsic motion, the intrinsic signal and extrinsic signal, respectively. We consider the intrinsic signal to be "noise" because it does not relate to extrinsic motion which we wish to detect and track.

This work is motivated by our inability to achieve the performance of $0.6 \mathrm{~m}$ average tracking error reported in [5] in a repeat of the identical experiment in May, 2010. Our new experiment was performed at the same location and using the identical hardware, number of nodes, and software. Yet, in the new experiment, variance-based radio tomographic imaging (VRTI) does not always locate the person walking inside the house as accurately as reported in [5]. Sometimes the position estimate error is as large as six meters, as shown in Figure 7. Investigation of the experimental data quickly indicates the reason for the degradation: periods of high wind. We first investigate the RSS measurements recorded during the calibration period, when no people are present inside the house. From the calibration measurements of [5], the variances are generally less than $5 \mathrm{~dB}$. However, the RSS measurements from our May 2010 experiment are quite variable, as shown in Figure 1. The RSS variance can be up to $30 \mathrm{~dB}$ in a short time window. Considering there is no person moving inside the house, that is, no extrinsic motion during the calibration period, the high variations of RSS measurements must be caused by intrinsic motion, in this case, wind-induced motion.

The variance caused by intrinsic motion can affect both model-based DFL and fingerprint-based DFL methods. To apply various DFL methods in practical applications, the intrinsic signal needs to be identified and removed or reduced. The subspace decomposition method has been used in spectral estimation, sensor array processing, and network anomaly 


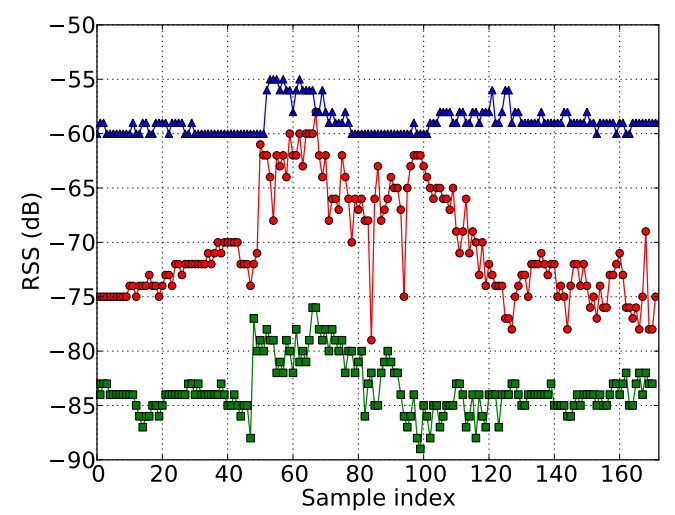

Fig. 1: Intrinsic signal measurements: RSS measurements from three links during the calibration period when no people are present in the environment.

detection [8], [9], [10], [11]. This method decomposes the measurement space into two subspaces, an intrinsic subspace and an extrinsic subspace. We find by projecting measurements onto the extrinsic subspace, the impact of intrinsic motion on a DFL system can be greatly reduced. In short, the changes due to intrinsic motion in the high-dimensional space of link measurements have a particular spatial signature, which we call an eigen-network, distinct from the changes due to human motion, which allows the intrinsic signal to be removed.

The contribution of this paper is to apply the subspace decomposition method to VRTI, which leads to a new algorithm we refer to as SubVRT. Experimental results show that SubVRT reduces the root mean squared error (RMSE) of the location estimate by $41 \%$ compared to VRTI. Further, we use Kalman filter to track people using localization estimates from VRTI and SubVRT. The cumulative distribution function (CDF) of the tracking errors shows that the tracking results from VRTI have $97 \%$ of errors less than $3.9 \mathrm{~m}$, while the tracking results from SubVRT have $97 \%$ of errors less than $1.4 \mathrm{~m}$, a $65 \%$ improvement.

The rest of this paper is organized as follows: Section II discusses the subspace decomposition method for noise reduction in DFL. Section III describes the experiments, Section IV shows the experimental results, and Section $\mathrm{V}$ investigates the Kalman filter tracking. Related work is presented in Section VI, and the conclusion is given in Section VII.

\section{Methods}

In this section, we first formulate a variance-based DFL problem, then we introduce PCA and the subspace decomposition method. Finally, we apply subspace decomposition to variance-based radio tomographic imaging, a new algorithm, subspace variance-based radio tomography (SubVRT).

\section{A. Problem statement}

For an RF sensor network with $N$ sensors (radio transceivers) deployed at static locations, we use $\mathbf{z}_{s, j}$ to denote the coordinate of sensor $j$. Since each sensor makes an RSS measurement with all other sensors, we use $s_{l, t}$ to denote the RSS measured at node $i_{l}$ sent by node $j_{l}$ at time $t$, where $i_{l}$ and $j_{l}$ are the receiver and transmitter number for link $l$, respectively. Time $t$ is discretized, thus $t \in \mathbb{Z}$. We assume constant transmitter power so that changes in $s_{l, t}$ are due to the channel, not to the transmitter. Then we denote the windowed RSS variance as:

$$
y_{l, t}=\frac{1}{m-1} \sum_{i=0}^{m-1}\left(\bar{s}_{l, t}-s_{l, t-i}\right)^{2}
$$

where $m$ is the length of the window, and $\bar{s}_{l, t}=$ $\frac{1}{m} \sum_{i=0}^{m-1} s_{l, t-i}$ is the sample average in this window period.

Consider that the network has $L$ directional links on which we measure signal strength (in general, $L \leq N(N-1)$ ). We let $\mathbf{y}=\left[y_{1}, y_{2}, \cdots, y_{L}\right]^{T}$ be the vector of windowed RSS variance from all $L$ links, and $\mathbf{y}^{(t)}=\left[y_{1, t}, y_{2, t}, \cdots, y_{L, t}\right]^{T}$ be the measurement vector $\mathbf{y}$ at time $t$. Then we use $\mathbf{y}_{c}$ to denote the calibration measurements collected during the calibration period, when no people are present in the environment; and we use $\mathbf{y}_{r}$ to denote the measurements from the real-time experiment period. The goal of DFL is to locate people during real-time operation. Our approach, as described in Section II-C, is to estimate an image map of the motion in the environment. From this map, we then infer the location of a person, as described in Section IV-B. First, we describe how the intrinsic signal can be reduced by subspace decomposition.

\section{B. Subspace decomposition method}

The subspace decomposition method has been widely used in spectral estimation, sensor array processing, etc. [8], [11] to improve estimation performance in noise. It is closely related to principal component analysis (PCA), which is widely used in finding patterns in high dimensional data [12].

From the $L$-dimensional calibration measurement vector $\mathbf{y}_{c}$, we may estimate its covariance matrix $C_{\mathbf{y}_{c}}$ as:

$$
C_{\mathbf{y}_{c}}=\frac{1}{M-1} \sum_{t=0}^{M-1}\left(\mathbf{y}_{c}^{(t)}-\boldsymbol{\mu}_{c}\right)\left(\mathbf{y}_{c}^{(t)}-\boldsymbol{\mu}_{c}\right)^{T}=A A^{T}
$$

where $M$ is the number of sample measurements, $\mathbf{y}_{c}^{(t)}$ is the calibration measurement vector $\mathbf{y}_{c}$ at time $t, \boldsymbol{\mu}_{c}=$ $\frac{1}{M} \sum_{t=0}^{M-1} \mathbf{y}_{c}^{(t)}$ is the sample average, and $A=\frac{1}{\sqrt{M-1}}\left[\mathbf{y}_{c}^{(0)}-\right.$ $\left.\boldsymbol{\mu}_{c}, \cdots, \mathbf{y}_{c}^{(M-1)}-\boldsymbol{\mu}_{c}\right]$ is an $L \times M$ matrix.

Instead of directly performing singular value decomposition (SVD) on $C_{\mathbf{y}_{c}}$, we perform SVD on an $M \times M$ matrix $A^{T} A$ [13]:

$$
A^{T} A \mathbf{v}_{i}=\gamma_{i} \mathbf{v}_{i}
$$

where $\mathbf{v}_{i}$ is the eigenvector corresponding to the eigenvalue $\gamma_{i}$. Right multiplying $A$ on both sides of (3), we obtain [13]:

$$
A A^{T} A \mathbf{v}_{i}=\gamma_{i} A \mathbf{v}_{i}
$$

From (4), we see $\mathbf{u}_{i}=A \mathbf{v}_{i}$ is the $i$ th eigenvector and $\gamma_{i}$ is the $i$ th eigenvalue of $C_{\mathbf{y}_{c}}$.

If the eigenvalues are in descending order, the first principal component $\mathbf{u}_{1}$ points in the direction of the maximum variance 
in the measurement, the second principal component $\mathbf{u}_{2}$ points in the direction of the maximum variance remaining in the measurement, and so on. If the first few eigenvalues are much larger than the others, then most of the variance in the measurements can be captured by these principal components.

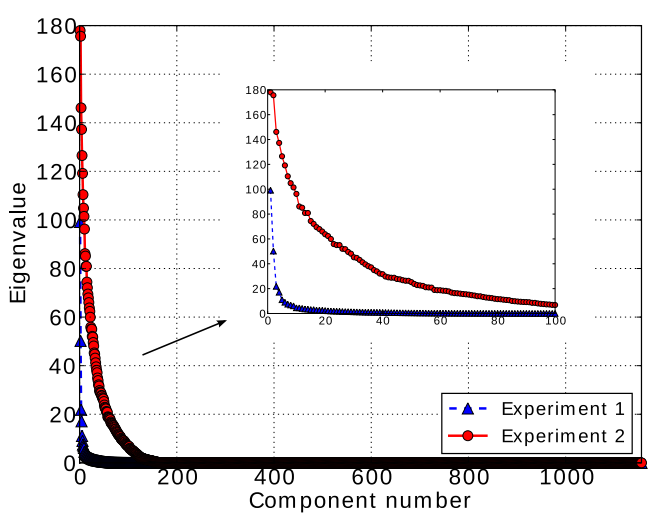

Fig. 2: Scree plot.

We perform PCA on calibration measurements from two sets of experiments as described in Section III. The eigenvalues of $C_{\mathbf{y}_{c}}$ from these experiments are shown in Figure 2. Because there is more intrinsic motion in Experiment 2, we see that the largest eigenvalue from Experiment 2 is almost twice as large as that from Experiment 1. We also see that for Experiment 1, the first four eigenvalues are much larger than the other eigenvalues, thus the corresponding eigenvectors can capture most of the variation in the measurements. However, for Experiment 2, there are more large-valued eigenvalues, and more eigenvectors are necessary to represent the major variation in the measurements. From the scree plot, we decide how many principal components, $k$, are necessary to capture the majority of the variations. Then, we use a lower dimensional space spanned by these principal components to represent the space containing the majority of the intrinsic signal measurements. This is the basic idea of the subspace decomposition method, which we discuss next.

In subspace decomposition, we divide all the principal components into two sets: $\hat{U}=\left[\mathbf{u}_{1}, \mathbf{u}_{2}, \cdots, \mathbf{u}_{k}\right]$ and $\tilde{U}=$ $\left[\mathbf{u}_{k+1}, \mathbf{u}_{k+2}, \cdots, \mathbf{u}_{L}\right]$. Then, we decompose the measurement space into two lower dimensional subspaces spanned by $\hat{U}$ and $\tilde{U}$. Since the variance during the calibration period is caused by intrinsic motion, that is, the variance captured by $\hat{U}$ is intrinsic signal, we call the subspace spanned by $\hat{U}$ the intrinsic subspace, and the other subspace spanned by $\tilde{U}$ the extrinsic subspace. Once the two subspaces are constructed, we can decompose the measurement vector $\mathbf{y}$ into two components - intrinsic signal component $\hat{\mathbf{y}}$ and extrinsic signal component $\tilde{\mathbf{y}}$ :

$$
\mathbf{y}=\hat{\mathbf{y}}+\tilde{\mathbf{y}}
$$

Since the principal components are orthogonal, the intrinsic signal component $\hat{\mathbf{y}}$ and the extrinsic signal component $\tilde{\mathbf{y}}$ can be formed by projecting $\mathbf{y}$ onto the intrinsic subspace and the extrinsic subspace, respectively:

$$
\begin{aligned}
& \hat{\mathbf{y}}=\Pi_{I} \mathbf{y}=\hat{U} \hat{U}^{T} \mathbf{y} \\
& \tilde{\mathbf{y}}=\Pi_{E} \mathbf{y}=\left(I-\hat{U} \hat{U}^{T}\right) \mathbf{y}
\end{aligned}
$$

where $\Pi_{I}=\hat{U} \hat{U}^{T}$ is the projection matrix for the intrinsic subspace, and $\Pi_{E}=I-\Pi_{I}$ is the projection matrix for the extrinsic subspace.

Using the procedures discussed above, we decompose the windowed RSS variance measurements $\mathbf{y}_{c}$ from the calibration period of Experiment 2 into intrinsic signal component $\hat{\mathbf{y}}_{c}$ and extrinsic signal component $\tilde{\mathbf{y}}_{c}$. We test a range of principal component number $k$ in constructing the intrinsic subspace. The decomposed intrinsic and extrinsic signal components for measurement on one link $l=588$ using different $k$ are shown in Figure 3. We see that if all principal components are used in constructing $\hat{U}$, that is, $k=L$, then $\hat{\mathbf{y}}_{c}$ is equivalent to the original measurement $\hat{\mathbf{y}}_{c}=\mathbf{y}_{c}$, while $\tilde{\mathbf{y}}_{c}$ is zero. If the first 100 principal components are used, since they capture most of the variance in the measurement, from Figure $3 \mathrm{~b}$ we see $\hat{\mathbf{y}}_{c}$ is almost the same as the original measurement. If we only use the first 40 eigenvectors, $\hat{\mathbf{y}}_{c}$ still matches the original measurement, as shown in Figure $3 \mathrm{a}$. That is, the first 40 principal components are sufficient to capture the majority of the variations in the measurements. Since each of the principal components used to construct the intrinsic subspace is an eigenvector of the covariance matrix of the network measurements, and each element in an eigenvector is from an individual link, we refer these eigenvectors as "eigennetworks".

In the above subspace decomposition derivation, we perform SVD on the matrix $A^{T} A$ instead of $A A^{T}$. For a network with $N$ sensors, there are $O\left(N^{2}\right)$ pairwise links, and the covariance matrix $C_{\mathbf{y}_{c}}$ is an $N^{2} \times N^{2}$ matrix. Since the computational complexity of performing SVD on a $L \times L$ matrix is $O\left(L^{3}\right)$ [14], that is, $O\left(N^{6}\right)$ for $C_{\mathbf{y}_{c}}$, directly performing SVD on high dimensional covariance matrix requires too much computation that increases quickly with $N$. Since the number of sample measurements is generally much lower than the number of links, $M<N^{2}$, this method greatly reduces the computational complexity of performing SVD from $O\left(N^{6}\right)$ to $O\left(M^{3}\right)$.

\section{SubVRT}

In VRTI, the presence of human motion within $P$ voxels of a physical space is denoted by $\mathbf{x}=\left[x_{1}, x_{2}, \ldots, x_{P}\right]^{T}$, where $x_{i}=1$ if motion occurs in voxel $i$, and $x_{i}=0$ otherwise. Work in [5] has shown the efficacy of a linear model that relates the motion image $\mathbf{x}$ to the RSS variance $\mathbf{y}_{r}$ :

$$
\mathbf{y}_{r}=W \mathbf{x}+\mathbf{n}
$$

where $\mathbf{n}$ is an $L \times 1$ noise vector, and $W$ is an $L \times P$ matrix representing the weighting of motion in each voxel on each link measurement. The weighting of voxel $p$ on link 


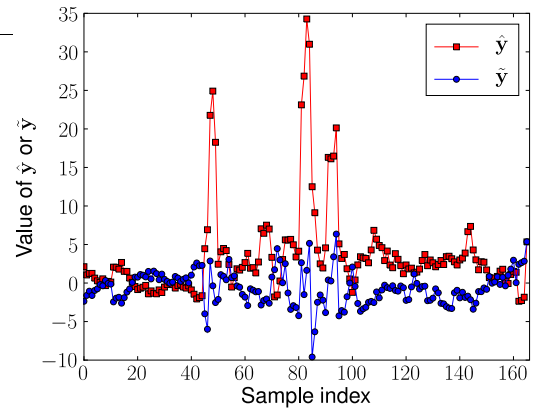

(a) $k=40$

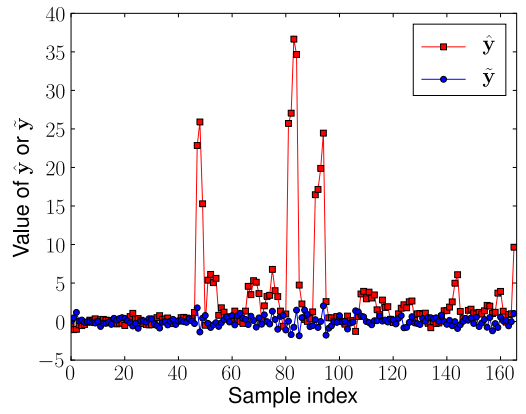

(b) $k=100$

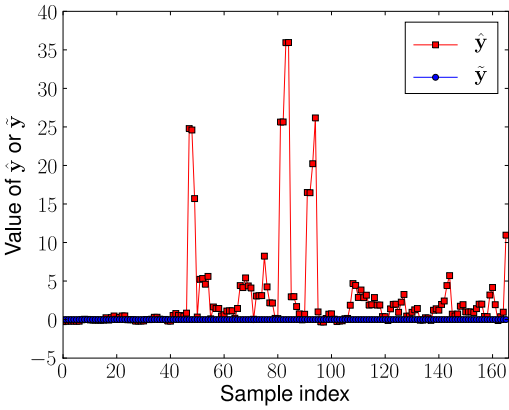

(c) $k=L$

Fig. 3: Effect of principal component number, $k$, on noise reduction. As $k$ increases, more of the measurement on link $l=588$ is attributed to noise, until at $k=L$, all of the measurement is considered to be noise.

$l$ is formulated as [5]:

$$
W_{l, p}=\frac{1}{\sqrt{d_{i_{l}, j_{l}}}} \begin{cases}\phi & \text { if } d_{i_{l}, p}+d_{j_{l}, p}<d_{i_{l}, j_{l}}+\lambda \\ 0 & \text { otherwise }\end{cases}
$$

where $d_{i_{l}, j_{l}}=\left\|\mathbf{z}_{s, i}-\mathbf{z}_{s, j}\right\|_{l_{2}}$ is the distance between two sensors $i, j$ on link $l$ located at $\mathbf{z}_{s, i}$ and $\mathbf{z}_{s, j}$ ( $l_{2}$ indicates the Euclidean norm); $d_{j_{l}, p}=\left\|\mathbf{z}_{s, j}-\mathbf{z}_{p}\right\|_{l_{2}}$ is the distance between $\mathbf{z}_{s, j}$, the location of sensor $j$ and $\mathbf{z}_{p}$, the center coordinate of voxel $p ; d_{i_{l}, p}=\left\|\mathbf{z}_{s, i}-\mathbf{z}_{p}\right\|_{l_{2}}$ is the distance between sensor $i$ and voxel $p ; \lambda$ is a tunable parameter controlling the radio ray width, and $\phi$ is a constant scaling factor normalizing the image. This model in (9) is an approximation that is shown to be effective in [5].

Once we have the forward model, the localization problem becomes an inverse problem: to estimate $P$ dimensional position vector $\mathrm{x}$ from $L$ dimensional link measurement vector $\mathbf{y}_{r}$. Certain regularization methods are necessary for this illposed inverse problem, and it is shown in [5] that submeter localization accuracy can be achieved by using the Tikhonov regularization. Thus, we use the Tikhonov regularized VRTI solution, which is given as:

$$
\hat{\mathbf{x}}=\Pi_{1} \mathbf{y}_{r} \quad \text { where } \Pi_{1}=\left(W^{T} W+\alpha Q^{T} Q\right)^{-1} W^{T}
$$

where $Q$ is the Tikhonov matrix, and $\alpha$ is a regularization parameter.

The key idea of SubVRT is to use the decomposed extrinsic signal component of the measurements in VRTI. We project the real-time measurement vector $\mathbf{y}_{r}$ onto the extrinsic subspace to obtain the extrinsic signal component $\tilde{\mathbf{y}}_{r}=$ $\left(I-\hat{U} \hat{U}^{T}\right) \mathbf{y}_{r}$. Then, we replace $\mathbf{y}_{r}$ in (10) with $\tilde{\mathbf{y}}_{r}$ and obtain the solution of SubVRT:

$$
\hat{\mathbf{x}}=\Pi_{2} \mathbf{y}_{r} \quad \text { where } \Pi_{2}=\left(W^{T} W+\alpha Q^{T} Q\right)^{-1} W^{T} \Pi_{E}
$$

From (11), we see that the solution is a linear transformation of the measurement vector. The transformation matrix $\Pi_{2}$ is the product of the transformation matrix $\Pi_{1}$ in (10) with the projection matrix for the extrinsic subspace $\Pi_{E}: \Pi_{2}=\Pi_{1} \Pi_{E}$.
Since the transformation matrix $\Pi_{2}$ does not depend on instantaneous real-time measurements, it can be pre-calculated, and it is easy to implement SubVRT for real-time applications.

We note that a major difference from VRTI is that SubVRT needs calibration, which results in $\Pi_{E}$ that is unique to the environment. However, calibration only requires that no extrinsic motion is present in the environment. In contrast to fingerprint-based DFL methods, SubVRT does not require training, i.e., that a person (or combinations of people) stands at all possible locations. Possible online calibration of SubVRT, and the trade-offs between calibration duration and localization accuracy, are left as future research topics.

\section{EXPERIMENTS}

We use measurements from two sets of experiments in this paper. We use the data set from the measurements conducted in March, 2009 reported by [5]. We call this data set Experiment 1 . The second experiment is a new experiment performed in May, 2010 at the same residential house, which we call Experiment 2. In both experiments, thirty-four TelosB nodes are deployed outside the living room of the house. As shown in Figure 5, eight nodes are placed on the table in the kitchen, six nodes are taped on boards extended outside the windows of the living room. The other twenty nodes are all placed on polyvinyl chloride (PVC) stands outside the house. All thirtyfour nodes are programmed with TinyOS program Spin [15], and a basestation connected to a laptop is used to collect pairwise RSS measurements from these nodes.

Both experiments are performed using the following procedure. Before people start to walk in the living room, a calibration is performed with no people (no extrinsic motion) in the experimental area. The duration of the calibration period of Experiment 1 is about 47 seconds, and 140 RSS link measurements are recorded, while for Experiment 2, 170 link measurements are recorded during a 57 seconds calibration period. Next, a person walks around a marked path in the living room at a constant speed, using a metronome and a metered path so that the position of the person at any particular time is known. In Experiments 1 and 2, a person walks four times 


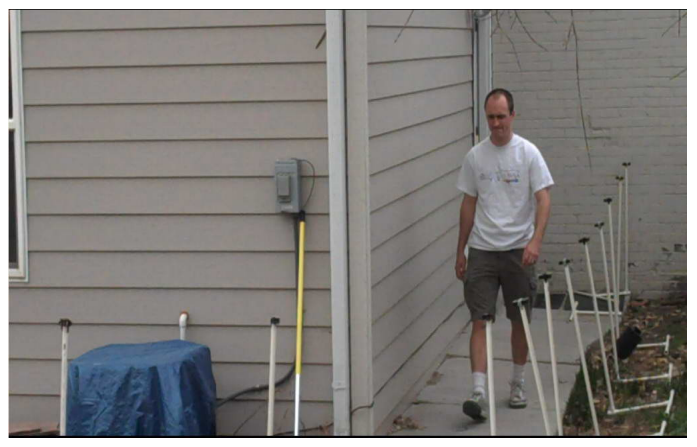

(a) Experiment 1

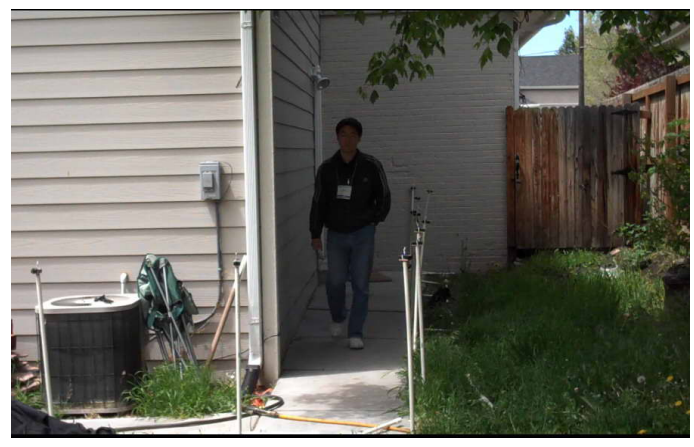

(b) Experiment 2

Fig. 4: Pictures of two experiments.

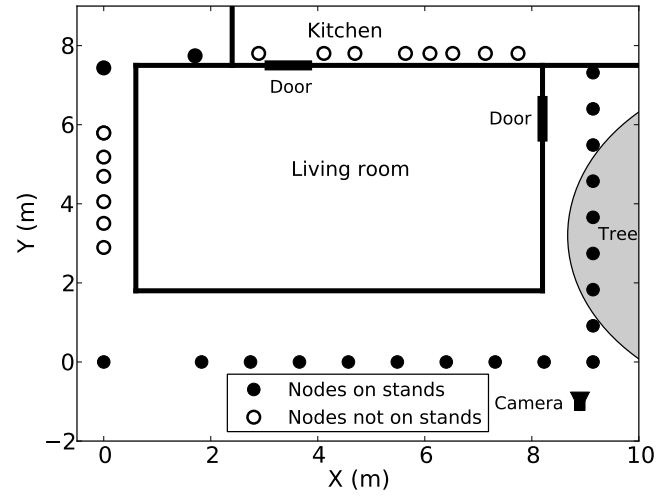

Fig. 5: Experimental layout of Experiment 2. The shade area is covered by tree branches and leaves.

around the squares shown in Figure 13(a) and (b), respectively.

These two through-wall experiments use the same hardware and software, and are performed following the same procedure. However, the main difference between these two experiments is the season. Experiment 1 is performed on a clear winter day, while Experiment 2 is performed on a windy day in late spring. As shown in Figure 4(a), there are no leaves on branches and no wind is observed from the video of Experiment 1. However, from the video recorded during Experiment 2 (one snapshot is shown in Figure 4(b)), we observe that wind causes grass, leaves and branches to sway. The wind also causes the PVC stands supporting the nodes to move. The swaying of leaves and branches and the movement of the PVC stands are intrinsic parts of the environment, which cannot be avoided, even when no people are present in the environment. Thus, the difference between Experiments 1 and 2 is that Experiment 2 has more intrinsic motion.

\section{REsults}

\section{A. Eigen-network results}

We use the calibration measurements from Experiment 2 to find the eigen-networks $\mathbf{u}_{i}$ as described in Section II-B. Since the first eigen-network $\mathbf{u}_{1}=\left[u_{11}, u_{12}, \cdots, u_{1 L}\right]^{T}$ points in the direction of the maximum variance of the calibration measurements $\mathbf{y}_{c}$, we show the first eigen-network $\mathbf{u}_{1}$ graphically, and proceed as follows. First, $\mathbf{u}_{1}$ elements are divided into three ranges: $0.2 \leq u_{1 l} / u_{\max }<0.3,0.3 \leq u_{1 l} / u_{\max }<0.4$, $0.4 \leq u_{1 l} / u_{\max }<1$, where $u_{\max }=\max _{l} u_{1 l}$. Then, a line is drawn between the transmitter sensor and receiver sensor coordinates for link $l$, with line type a function of which range $u_{1 l}$ is within. This is shown in Figure 6.

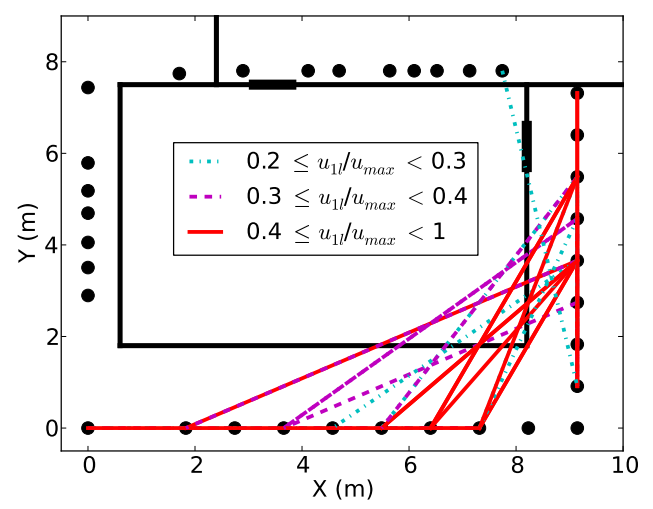

Fig. 6: Plot of the first eigen-network $\mathbf{u}_{1}$ (links $l$ are plotted with different line types based on their $u_{1 l}$ values).

We see the links with $u_{1 l}$ values higher than $0.4 u_{\max }$ are all in the lower right side of the house. This is consistent with our observation that the intrinsic motion of the movement of leaves and branches on the tree located to the right side of the house cause significant variations in the RSS measured on links likely to have RF propagation through the branches and leaves. Note that links with high $u_{1 l}$ values all have at least one end point near the tree. In particular, links which are likely to see significant diffraction around the bottom-right corner of the house have high $u_{1 l}$ values. The leaves and branches almost touch this corner, as seen in Figure 4(b). Not only do these links measure high RSS variance during the calibration period, they do so simultaneously. That is, the fact that these links have high positive $u_{1 l}$ values indicates that when one of these links experiences increased RSS variance, the other links also measure increased RSS variance. Thus, the first eigen-network 
$\mathbf{u}_{1}$ becomes a spatial signature for intrinsic motion-induced RSS variance. When we see this linear combination in $\mathbf{y}_{r}$, we should attribute it to intrinsic, rather than extrinsic motion. These observations about the source of RSS variance on links support the idea that intrinsic motion in the environment causes increased RSS variance simultaneously on multiple links.

\section{B. Localization results}

Now, we evaluate VRTI and SubVRT using measurements from Experiments 1 and 2. From VRTI and SubVRT, we obtain reconstructed motion images, the position of the moving person can be estimated by finding the center coordinate of the voxel with maximum value. Thus, a VRTI or SubVRT localization estimate is defined as:

$$
\hat{\mathbf{z}}=\mathbf{z}_{q} \quad \text { where } \quad q=\underset{p}{\arg \max } \hat{x}_{p}
$$

where $\mathbf{z}_{q}$ is the center coordinate of voxel $q$, and $\hat{x}_{p}$ is the $p$ th element of vector $\hat{\mathbf{x}}=\left[\hat{x}_{1}, \hat{x}_{2}, \ldots, \hat{x}_{P}\right]^{T}$ from (10) or (11). Then, the localization error is defined as: $e_{l o c}=\|\hat{\mathbf{z}}-\mathbf{z}\|_{l_{2}}$, where $\mathbf{z}$ is the actual position of the person.

The VRTI estimates of Experiment 2 are shown in Figure 7. For clarity, we only show the actual/estimated positions when the person walks the last round of the square. We find that due to the impact of intrinsic motion, some estimates of VRTI are greatly biased to the right side of the experimental area (i.e., five estimates with more than $4 \mathrm{~m}$ error, as shown in Figure 7). However, for SubVRT, the impact of intrinsic motion is greatly reduced. As shown in Figure 8, the estimates from SubVRT are more accurate than VRTI. There are no estimate errors larger than $2 \mathrm{~m}$. Note that for both algorithms, two estimates are outside the house. The algorithms presented do not include any prior information of the house map or physical barriers which would prevent certain trajectories. Incorporation of prior knowledge of an indoor environment might be used to obtain better estimates, but at the expense of requiring more information to deploy the system.

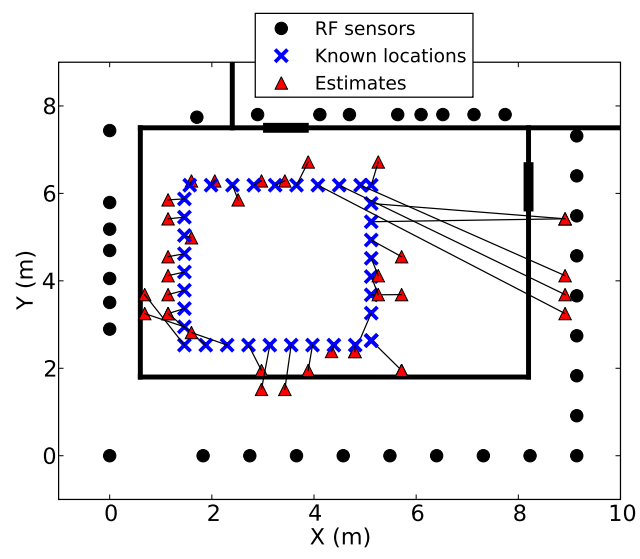

Fig. 7: Estimates from VRTI.

Quantatively, we compare the localization errors from VRTI and SubVRT for the full data set in Figure 9. We see that there are several periods during which the localization errors

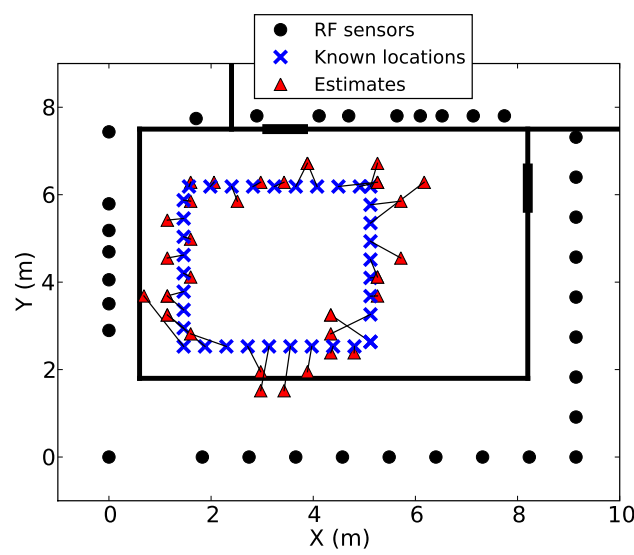

Fig. 8: Estimates from SubVRT.

\begin{tabular}{|c|ccc|}
\hline RMSE (meter) & VRTI & SubVRT & Improvement \\
\hline Experiment 1 & 0.70 & 0.65 & $7.0 \%$ \\
\hline Experiment 2 & 1.26 & 0.74 & $41.3 \%$ \\
\hline
\end{tabular}

TABLE I: Localization RMSEs from VRTI and SubVRT.

from VRTI are several orders higher than those from SubVRT. Specifically, the localization errors during a period with high wind, from sample index 205 to 221, are shown in the inset of Figure 9. During this period, the average localization error from VRTI is $3.0 \mathrm{~m}$, while the average error from SubVRT is only $0.62 \mathrm{~m}$, a $79 \%$ improvement. We also compare the RMSE of the estimates, which is defined as the square root of the average squared localization error over the course of the entire experiment. The RMSEs from the two experiments are summarized in Table I. For Experiment 1, the RMSE from VRTI is $0.70 \mathrm{~m}$, while the RMSE from SubVRT is $0.65 \mathrm{~m}$. Since there is not much intrinsic motion in Experiment 1, the improvement in RMSE from SubVRT is 7.0\%. For Experiment 2, the RMSE from VRTI is $1.26 \mathrm{~m}$, compared to $0.74 \mathrm{~m}$ for SubVRT, a $41.3 \%$ improvement.

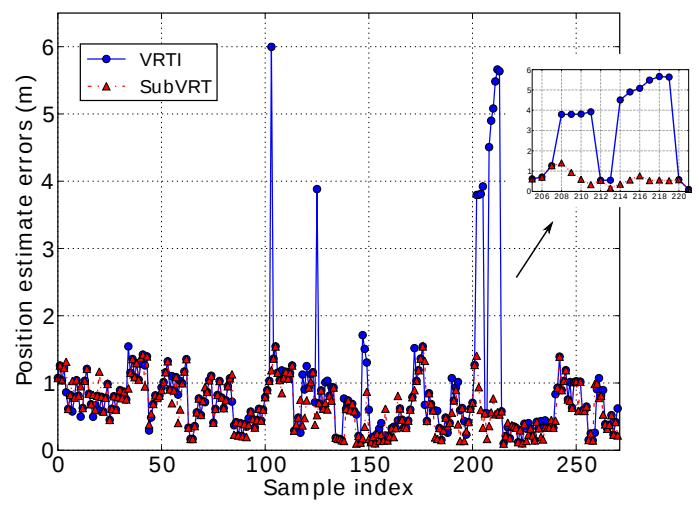

Fig. 9: Estimate errors from VRTI and SubVRT. 


\section{Discussion}

The parameters that we use in VRTI and SubVRT are listed in Table II. Here, we discuss the effects of the regularization parameter $\alpha$, widow length $m$, and the number of principal components $k$ on the SubVRT localization results.

Since SubVRT is an ill-posed inverse problem, the regularization parameter $\alpha$ plays an important role. The RMSE of Experiment 2 using a range of regularization parameters is shown in Figure 10. We see that $\alpha$ is a trade-off between the prior knowledge (smoothness of the image) and the measurements. When $\alpha$ is between 50 and 100, the RMSE reaches the minimum. From Figure 10, we can also see the effects of window length $m$ used in calculating the windowed variance. For $m=2$, too few samples are used to calculate the variance, as the RMSE is always above $1 \mathrm{~m}$. On the other hand, if $m$ is too large, i.e., $m=8$, then the variance calculated from a long window history may introduce unacceptable delay for locating fast-moving people. Thus, the window length parameter $m$ is a trade-off between sufficient samples and latency. For the two experiments, we choose $m=4$.

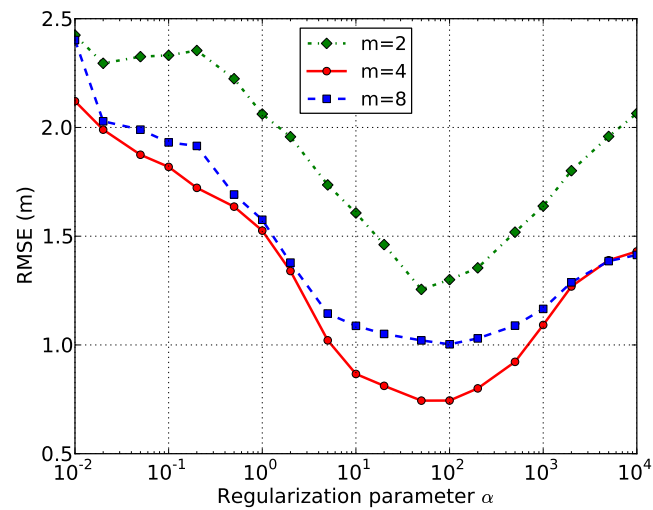

Fig. 10: Localization RMSE vs. regularization parameter $\alpha$.

Another important parameter for SubVRT is the number of principal components used to construct the intrinsic subspace. As discussed in Section II-B, the first $k$ components are used to calculate the projection matrix for the intrinsic subspace $\Pi_{I}$. If $k=0, \Pi_{I}=0$, then $\Pi_{1}=\Pi_{2}$, SubVRT is simplified to VRTI. The RMSE of SubVRT using a range of $k$ are shown in Figure 11. Since the first eigen-network $\mathbf{u}_{1}$ captures the strongest intrinsic signal, when $k=1$, the RMSE of Experiment 2 decreases substantially from $1.26 \mathrm{~m}$ to $0.82 \mathrm{~m}$. Since Experiment 1 has less intrinsic motion, the RMSE decreases from $0.70 \mathrm{~m}$ when $k=0$ to $0.65 \mathrm{~m}$ when $k=4$, a less substantial decrease. We note that as $k$ increases, more and more information in the measurement is removed, and the RMSE stops decreasing dramatically, and even increases, at certain $k$. This is because when $k$ becomes very large, the information removed also contains a great amount of signal caused by extrinsic (human) motion. Thus, the performance of SubVRT could be degraded if $k$ is chosen to be too large. The parameter $k$ is a trade-off between removing intrinsic motion impact and keeping useful information from extrinsic motion. For experiments without much intrinsic motion, such as Experiment 1, we choose a small $k$. However, for Experiment 2, with strong impact from intrinsic motion, we use a large $k$. As listed in Table II, we use $k=4$ and $k=36$ for Experiments 1 and 2, respectively. Algorithms to determine $k$ automatically, regardless of the environment, will require more experiments in a variety of situations, which will require future work.

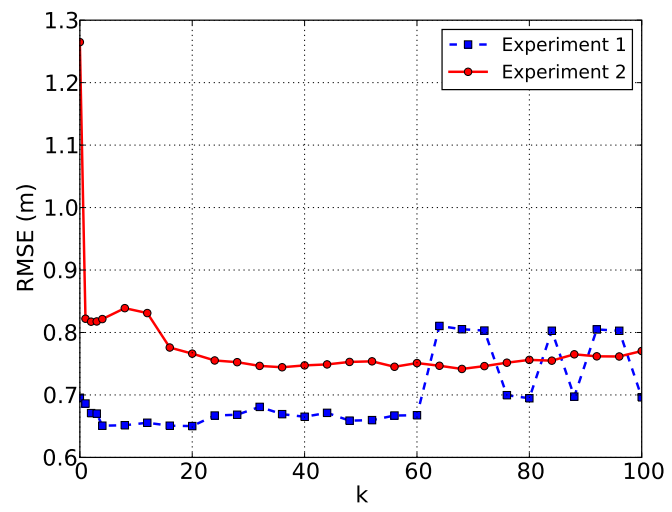

Fig. 11: Localization RMSE vs. principal component number $k$.

\section{TRACKING}

In this section, we apply a Kalman filter to the localization estimates shown in Section IV-B to better estimate moving people's positions over time. Then, we compare the tracking results from VRTI and SubVRT, and show that the Kalman filter tracking results from SubVRT are more robust to large localization errors.

\section{A. Kalman filter}

In the state transition model of the Kalman filter, we include both position $\left(P_{x}, P_{y}\right)$ and velocity $\left(V_{x}, V_{y}\right)$ in the Cartesian coordinate system in the state vector $\mathbf{s}=\left[P_{x}, P_{y}, V_{x}, V_{y}\right]^{T}$, and the state transition model is:

$$
\mathbf{s}[t]=G \mathbf{s}[t-1]+\mathbf{w}[t]
$$

where $\mathbf{w}=\left[0,0, w_{x}, w_{y}\right]^{T}$ is the process noise, and $G$ is:

$$
G=\left[\begin{array}{llll}
1 & 0 & 1 & 0 \\
0 & 1 & 0 & 1 \\
0 & 0 & 1 & 0 \\
0 & 0 & 0 & 1
\end{array}\right]
$$

The observation inputs $\mathbf{r}[t]$ of the Kalman filter are the localization estimates from VRTI or SubVRT at time $t$, and the observation model is:

$$
\mathbf{r}[t]=H \mathbf{s}[t]+\mathbf{v}[t]
$$

where $\mathbf{v}=\left[v_{x}, v_{y}\right]^{T}$ is the measurement noise, and $H$ is:

$$
H=\left[\begin{array}{llll}
1 & 0 & 0 & 0 \\
0 & 1 & 0 & 0
\end{array}\right]
$$




\begin{tabular}{|c|c|c|}
\hline Parameter & Value & Description \\
\hline$\alpha$ & 100 & Regularization parameter \\
\hline$m$ & 4 & Window length to calculate variance \\
\hline$k$ & $4 / 36$ & Number of principal components \\
\hline$\sigma_{w}^{2}$ & 2 & Process noise parameter \\
\hline$\sigma_{v}^{2}$ & 5 & Measurement noise parameter \\
\hline
\end{tabular}

TABLE II: Parameters in VRTI, SubVRT, and Kalman filter.

\begin{tabular}{|c|ccc|}
\hline RMSE (meter) & VRTI & SubVRT & Improvement \\
\hline Experiment 1 & 0.66 & 0.57 & $13.6 \%$ \\
\hline Experiment 2 & 1.21 & 0.72 & $40.5 \%$ \\
\hline
\end{tabular}

TABLE III: Tracking RMSEs from VRTI and SubVRT.

In the Kalman filter, $v_{x}$ and $v_{y}$ are zero-mean Gaussian with variance $\sigma_{v}^{2}, w_{x}$ and $w_{y}$ are zero-mean Gaussian with variance $\sigma_{w}^{2}$ [16]. The parameters $\sigma_{v}^{2}$ and $\sigma_{w}^{2}$ of the measurement noise and process noise are listed in Table II.

\section{B. Tracking results}

We use the Kalman filter described above to track the positions of the person. The cumulative distribution functions (CDFs) of the tracking errors from Experiment 2 are shown in Figure 12. We see that the Kalman filter tracking results from VRTI have many more large errors than SubVRT. $97 \%$ of the tracking errors from VRTI are less than $3.9 \mathrm{~m}$, while $97 \%$ of the tracking errors from SubVRT are less than $1.4 \mathrm{~m}$, a $65.3 \%$ improvement. We use the $97^{\text {th }}$ percentile of errors to show the robustness of the tracking algorithm to large errors, and the CDF shows the tracking results from SubVRT are more robust to these large errors.

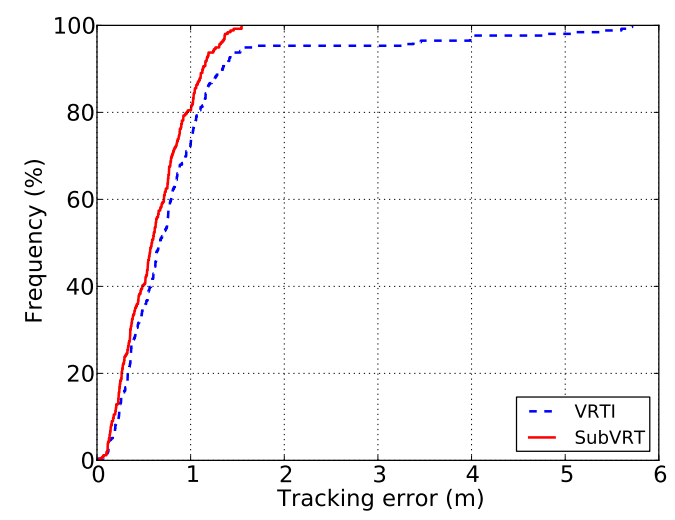

Fig. 12: CDFs of tracking errors.

We also compare the RMSE of the tracking results from VRTI and SubVRT, which are listed in Table III. For Experiment 1, the tracking RMSE from SubVRT is $0.57 \mathrm{~m}$, a $13.6 \%$ improvement compared to the RMSE of $0.66 \mathrm{~m}$ from VRTI. For Experiment 2, the tracking RMSE from SubVRT is reduced by $40.5 \%$ to $0.72 \mathrm{~m}$ compared to $1.21 \mathrm{~m}$ RMSE from VRTI. We note that the tracking RMSEs from VRTI and SubVRT of Experiment 2 are both larger than Experiment 1 due to the impact of intrinsic motion. However, for VRTI the tracking RMSE from Experiment 2 has a $83.3 \%$ increase compared to Experiment 1, while for SubVRT, it only increases $26.3 \%$. The tracking RMSE from SubVRT is more robust to the impact of intrinsic motion.

Finally, the Kalman filter tracking results from SubVRT of Experiments 1 and 2 are shown in Figure 13(a) and (b), respectively. Even during Experiment 2, with significant intrinsic motion, the SubVRT algorithm with Kalman filter can still track a person with submeter accuracy.

(a)
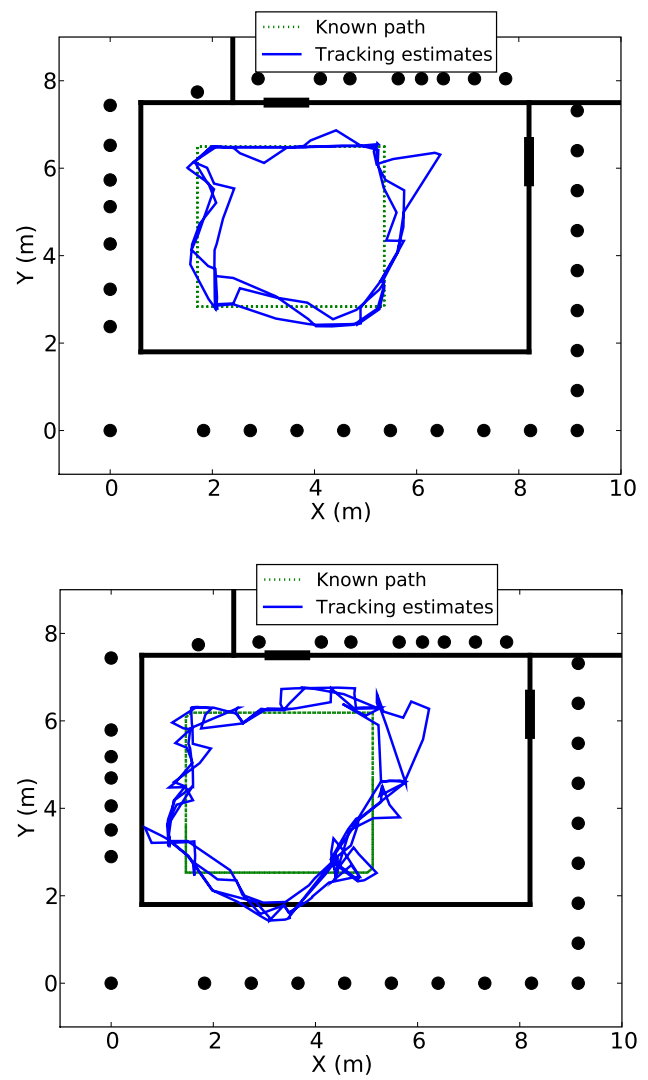

(b)

Fig. 13: Kalman filter tracking results from SubVRT. (a) Tracking results of Experiment 1; (b) Tracking results of Experiment 2.

\section{Discussion}

In the Kalman filter, the process noise parameter $\sigma_{w}^{2}$ should be chosen according to the dynamics of the movement. For example, for tracking vehicles, $\sigma_{w}^{2}$ should be set to a large value. The measurement noise parameter $\sigma_{v}^{2}$ depends on how accurate the observation inputs are. Here, we choose $\sigma_{w}^{2}$ based on the speed of moving people in typical homes, and we test the effect of using different $\sigma_{v}^{2}$ on the tracking errors. The tracking RMSEs from SubVRT for Experiments 1 and 2 are shown as functions of $\sigma_{v}^{2}$ in Figure 14. If $\sigma_{v}^{2}$ is too large, the Kalman filter gives very small weights to observation inputs. On the other hand, for very small measurement noise parameter, the system dynamic model contributes little to the Kalman filter. Thus, the RMSE reaches the minimum when an appropriate balance between observation inputs and dynamic 
model is found. We also note from Figure 14 that for both Experiments, the RMSEs are shallow functions of $\sigma_{v}^{2}$ in a wide range from 0.001 to 20 . That is, if we give sufficient weights to the observation inputs, which are the localization estimates from SubVRT, our Kalman filter tracking results are not very sensitive to the measurement noise parameter.

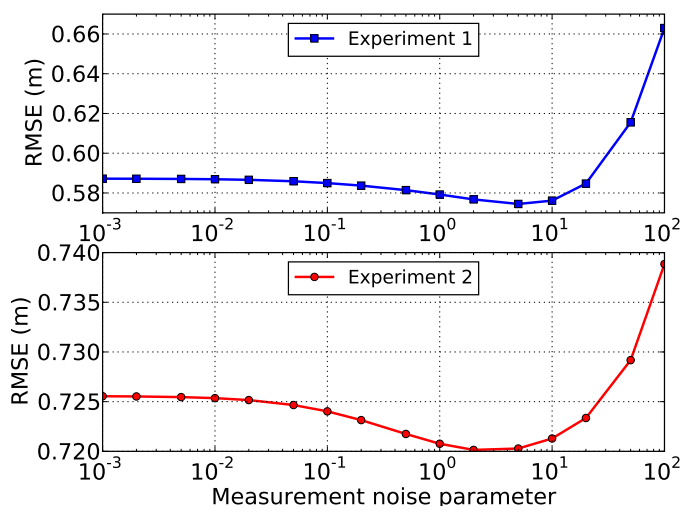

Fig. 14: Tracking RMSE vs. measurement noise parameter $\sigma_{v}^{2}$.

\section{RELATED WORK}

DFL using RF sensor networks has potential applications in surveillance for police and firefighters. Different measurements and algorithms have been proposed [4], [6], [7], [5]. For RSSbased DFL, there are essentially two types of algorithms: fingerprint-based algorithms and model-based algorithms. Like fingerprint-based real-time location service (RTLS) systems, fingerprint-based DFL methods use a database of training measurements, in which a person stands at all possible locations, and estimate people's locations by comparing the measurements during the online phase with the training measurements [6], [7], [17]. Since a separate training measurement dataset is necessary, fingerprint-based DFL needs substantial calibration effort. As the number of people to be located increases, the training requirement increases exponentially. Model-based algorithms [5], [18], [19] provide another approach. A forward model is used to relate measurements with unknown people's positions, and the localization problem can be solved as an inverse problem. An advantage of a model-based algorithm is that it does not need such training measurements, however, sufficient link measurements are necessary to solve the inverse problem. The proposed subspace decomposition method has been applied to a model-based DFL method - VRTI, and can significantly improve the robustness of position estimates. The subspace decomposition method may also be used in fingerprint-based DFL methods, but we leave this as a possible future research topic.

\section{CONCLUSION}

In this paper, we propose to use the subspace decomposition method to reduce noise for RSS variance-based device-free localization and tracking. We discuss that intrinsic motion, such as moving leaves, increase measured RSS variance, in a way that is "noise" to a DFL system. The signal caused by intrinsic motion has a spatial signature, which can be removed by the proposed method. We apply the subspace decomposition method to VRTI, a new algorithm we call SubVRT. Experimental results show that SubVRT can reduce localization RMSE by $41 \%$. We further apply a Kalman filter on SubVRT estimates for tracking. We find the tracking results from SubVRT are significantly more robust to large errors.

\section{ACKNOWLEDGMENT}

The authors would like to thank Joey Wilson for assisting with the experiments and Sai Ananthanarayanan for his edits.

\section{REFERENCES}

[1] N. Patwari and J. Wilson, "RF sensor networks for device-free localization: Measurements, models and algorithms," Proceedings of the IEEE, vol. 98, no. 11, pp. 1961-1973, Nov. 2010.

[2] C. Chang and A. Sahai, "Object tracking in a 2D UWB sensor network," in 38th Asilomar Conference on Signals, Systems and Computers, vol. 1, Nov. 2004, pp. 1252-1256.

[3] L.-P. Song, C. Yu, and Q. H. Liu, "Through-wall imaging (TWI) by radar: 2-D tomographic results and analyses," IEEE Transactions on Geoscience and Remote Sensing, vol. 43, no. 12, pp. 2793-2798, Dec. 2005.

[4] M. Youssef, M. Mah, and A. Agrawala, "Challenges: device-free passive localization for wireless environments,' in MobiCom '07: ACM Int'l Conf. Mobile Computing and Networking, 2007, pp. 222-229.

[5] J. Wilson and N. Patwari, "See-through walls: Motion tracking using variance-based radio tomography networks," IEEE Transactions on Mobile Computing, vol. 10, no. 5, pp. 612-621, May 2011.

[6] D. Zhang, J. Ma, Q. Chen, and L. M. Ni, "An RF-based system for tracking transceiver-free objects," in IEEE PerCom'07, 2007, pp. 135144.

[7] F. Viani, L. Lizzi, P. Rocca, M. Benedetti, M. Donelli, and A. Massa "Object tracking through RSSI measurements in wireless sensor networks," IEE Electronics Letters, vol. 44, no. 10, pp. 653-654, 2008.

[8] P. Stoica and R. L. Moses, Introduction to Spectral Analysis. New Jersey: Prentice-Hall Inc., 1997.

[9] R. Schmidt, "Multiple emitter location and signal parameter estimation," IEEE Transactions on Antennas and Propagation, vol. 34, pp. 276-280, March 1986.

[10] R. Roy and T. Kailath, "ESPRIT - estimation of signal parameters via rotational invariance techniques," IEEE Transactions on Acoustics, Speech, and Signal Processing, vol. 37, no. 7, pp. 984-995, July 1989.

[11] A. Lakhina, M. Crovella, and C. Diot, "Diagnosing network-wide traffic anomalies," in ACM SIGCOMM, Aug. 2004.

[12] I. Jolliffe, Principal Component Analysis, 2nd ed. Springer-Verlag New York, 2002.

[13] M. Turk and A. Pentland, "Eigenfaces for recognition," Journal of Cognitive Neuroscience, vol. 3, no. 1, pp. 71-86, 1991.

[14] G. Xu and T. Kailath, "Fast subspace decomposition," IEEE Transactions on Signal Processing, vol. 42, no. 3, pp. 539-551, March 1994.

[15] Sensing and Processing Across Networks (SPAN) Lab Spin website. http://span.ece.utah.edu/spin.

[16] S. M. Kay, Fundamentals of Statistical Signal Processing. New Jersey: Prentice Hall, 1993.

[17] M. Seifeldin and M. Youssef, "Nuzzer: A large-scale device-free passive localization system for wireless environments," Arxiv.org, Tech. Rep. arXiv:0908.0893, Aug. 2009.

[18] J. Wilson and N. Patwari, "Radio tomographic imaging with wireless networks," IEEE Transactions on Mobile Computing, vol. 9, no. 5, pp. 621-632, May 2010.

[19] M. A. Kanso and M. G. Rabbat, "Compressed RF tomography for wireless sensor networks: Centralized and decentralized approaches," in 5th IEEE Intl. Conf. on Distributed Computing in Sensor Systems (DCOSS-09), Marina Del Rey, CA, June 2009. 